\title{
ON THE EXTENSIONS OF INFINITE-DIMENSIONAL REPRESENTATIONS OF LIE SEMIGROUPS
}

\author{
ADOLF R. MIROTIN
}

Received 22 January 2001

\begin{abstract}
The necessary and sufficient conditions have been obtained for extendability of a Banach representation of a generating Lie semigroup $S$ to a local representation of the Lie group $G$ generated by $S$ when the tangent wedge of $S$ is a Lie semialgebra. The most convenient conditions we obtain correspond to the case of unitary representations. In this case, we give a criterion of global extendability if $G$ is exponential and solvable.
\end{abstract}

2000 Mathematics Subject Classification: 20M30, 22E99, 43A65.

1. Introduction. If $S$ is a subsemigroup of a topological group $G$ with interior points and $G$ is a left quotient group for $S$, it is easy to prove that every representation of $S$ by invertible operators on a Banach space $\mathscr{V}$ may be extended, in a unique manner, to the representation of $G$ on $\mathscr{V}$ (see Proposition 6.1 below). It is easy to prove that every finite-dimensional representation of a generating Lie semigroup $S$ can be extended to the local representation of the Lie group $G$ generated by $S$, if $G$ is connected and the tangent wedge of $S$ is a Lie semialgebra [14]. In this paper, using the infinitesimal method, we study the problem of extendability of a Banach representations $\pi$ of a generating Lie semigroup $S$ to the connected Lie group $G$ generated by $S$ when the tangent wedge of $S$ is a Lie semialgebra. We show that the differential $d \pi$ extends to the representation of the Lie algebra of $G$ if at least one operator $\pi\left(s_{0}\right), s_{0} \in \operatorname{int} S$, is invertible. In this way, the necessary and sufficient conditions of a local extendability of $\pi$ have been obtained in terms of the tangent objects. The most convenient conditions we obtain, correspond to the case of unitary representations. Thus, the problem of extendability of a representation of $S$ to $G$ has been reduced to the (group theoretical) problem of extendability of a local representation of $G$ to a global one (see Corollary 2.2 below). For exponential and solvable $G$, we give also necessary and sufficient conditions of global extendability of unitary representations.

The main results of this paper appeared earlier in [12]. One-dimensional and positive representations of $S$ were studied in detail in [13].

Throughout, unless otherwise stated, $G$ denotes a connected Lie group with unit $e, L(G)$ its Lie algebra, and exp : $L(G) \rightarrow G$ its exponential function. For a closed subsemigroup $S$ of $G$ its tangent wedge is defined by

$$
L(S):=\left\{X \in L(G): \exp \left(\mathbb{R}^{+} X\right) \subseteq S\right\} .
$$

A closed subsemigroup $S \subseteq G$ is called Lie semigroup if $S$ is the closure in $G$ of $\langle\exp L(S)\rangle$, the semigroup, generated by

$$
\exp L(S):=\{\exp X: X \in L(S)\} .
$$


After shrinking $G$ we may assume that $S$ is a generating Lie semigroup, that is, $L(G)$ is the smallest Lie algebra containing $L(S)$ (see [6]).

DEFINITION 1.1. A Lie subsemigroup $S \subseteq G$ is quasi-invariant if its tangent wedge $L(S)$ is a Lie semialgebra, that is, for some Campbell-Hausdorff-neighborhood $B$ in $L(G)$

$$
(L(S) \cap B) *(L(S) \cap B) \subseteq L(S),
$$

where $*$ denotes the Campbell-Hausdorff multiplication in $L(G)$

$$
X * Y=(X+Y)+\frac{1}{2}[X, Y]+\cdots+H_{n}(X, Y)+\cdots .
$$

Note that every invariant (with respect to all inner automorphisms of the group $G$ ) generating Lie semigroup $S$ is quasi-invariant [16, Proposition IV.7], [6, Theorem III.2.15], but the converse is false. As an example, take the subsemigroup $S^{+} \subset \mathrm{GL}(2, \mathbb{R})$ consisting of all matrices with nonnegative entries [8, Example 6.3, page 182].

For a generating Lie semigroup $S$ in a connected Lie group $G$ the interior int $S$ of $S$ (with respect to $G$ ) is a dense ideal of $S$, and $\operatorname{int} S \subseteq\langle\exp L(S)\rangle$ [16, Proposition IV.6], [6, Theorem V.1.10]. If, in addition, $S$ is quasi-invariant then, according to [6, Theorem II.2.13], we have

$$
L(G)=L(S)-L(S) .
$$

The basic reference in Lie semigroups is [6].

We will employ some preliminary results to prove our main theorems.

2. The Gäding space. Let $\pi$ be a representation of $S$ on a Banach space $\mathscr{V}$, that is, a homomorphism of $S$ into the multiplicative semigroup $\mathrm{gl}(\mathscr{V})$ of all bounded (linear) operators on $\mathscr{V}$ which is continuous with respect to the strong operator topology and satisfies $\pi(e)=I$, the identity operator on $\mathscr{V}$. For every $X \in L(S)$ the map $t \mapsto$ $\pi(\exp t X): \mathbb{R}^{+} \rightarrow \mathrm{gl}(\mathscr{V})$ is a one-parameter $C_{0}$-semigroup of operators. We denote by $A(X)$ or $d \pi(X)$ the generator of this semigroup.

LEMMA 2.1. The representation $\pi$ of a Lie semigroup $S$ is uniquely determined by $d \pi$.

Proof. Let $\pi$ and $\pi_{1}$ be two representations of $S$ in a Banach space $\mathscr{V}$ and $d \pi(X)=$ $d \pi_{1}(X)$ for all $X \in L(S)$. Then $C_{0}$-semigroups $t \mapsto \pi(\exp t X)$ and $t \mapsto \pi_{1}(\exp t X)$ coincide $(X \in L(S))$. Therefore, $\pi\left|\langle\exp L(S)\rangle=\pi_{1}\right|\langle\exp L(S)\rangle$, and $\pi=\pi_{1}$ by continuity.

COROLLARY 2.2. Let a Banach representation $\pi$ of a Lie semigroup $S$ extend to a local representation $T$ of the Lie group $G$ generated by $S$. If, in turn, $T$ extends to a representation $\hat{\pi}$ of the whole $G$, then $\hat{\pi}$ is an extension of $\pi$ to $G$.

Indeed, set $\pi_{1}:=\hat{\pi} \mid S$. Then $\pi_{1}$ and $\pi$ coincide on the set $U \cap S$, where $U$ is an $e$-neighborhood in $G$, and so $d \pi_{1}=d \pi$.

Let $C_{0}^{\infty}(S)$ be the space of all compactly supported functions in $C^{\infty}(G)$ concentrated in int $S$. For $\phi \in C_{0}^{\infty}(S)$ and $u \in \mathscr{V}$, set

$$
u(\phi):=\int_{G} \phi(x) \pi(x) u d_{l} x
$$


( $d_{l} x$ denotes the left Haar measure in $G$ ), and denote by $\mathscr{D}_{S}$ the linear subspace of $\mathscr{V}$ generated by $\left\{u(\phi): \phi \in C_{0}^{\infty}(S), u \in \mathscr{V}\right\}$. For the case $S=G$ we get the Garding space $\mathscr{D}_{G}$ (cf. [1, Chapter 11, Section 1]). We denote also by $\mathscr{V}^{\infty}$ the set of such $u \in \mathscr{V}$ that the function $s \mapsto \pi(s) u$ is in $C^{\infty}(\operatorname{int} S)$.

Proposition 2.3. Let $S$ be a quasi-invariant generating Lie semigroup in a connected Lie group $G$ and let $\pi$ be a representation of $S$ on a Banach space $\mathscr{V}$. Then the following assertions hold:

(i) $\mathscr{D}_{S} \subseteq \mathscr{V}^{\infty}$.

(ii) $\mathscr{D}_{S} \subseteq \mathscr{D}(A(X))$, the domain of $A(X)$, and $\mathscr{D}_{S}$ is $A(X)$-invariant and $\pi$-invariant $(X \in L(S))$.

(iii) $\mathscr{D}_{S}$ is dense in $\mathscr{V}$.

(iv) The map $X \mapsto A(X) \mid \mathscr{D}_{S}$ extends uniquely to a linear mapping $\hat{A}$ between $L(G)$ and the linear space of operators on $\mathscr{D}_{S}$.

(v) If $\pi\left(s_{0}\right)$ is invertible for some $s_{0} \in \operatorname{int} S$, then $\mathscr{V}^{\infty} \subseteq \mathscr{D}(A(X))(X \in L(S))$, and the map $X \mapsto A(X) \mid \mathscr{V}^{\infty}$ extends uniquely to a linear mapping $\hat{A}$ between $L(G)$ and the linear space of operators $\mathscr{V}^{\infty} \rightarrow \mathscr{V}$.

Proof. (i) Following the proof of Theorem 1 in [1, Chapter 11], let $y(t)=\exp t Y$ $\left(Y \in L(G), t \in \mathbb{R}\right.$ ) be a one-parameter subgroup in $G, \phi \in C_{0}^{\infty}(S), s_{0} \in \operatorname{int} S$. Then (cf. [1, formula (14)])

$$
t^{-1}\left(\pi\left(s_{0} y(t)\right)-\pi\left(s_{0}\right)\right) u(\phi)=\pi\left(s_{0}\right) \int_{G} t^{-1}\left(\phi\left(y^{-1}(t) x\right)-\phi(x)\right) \pi(x) u d_{l} x .
$$

Setting in (2.2) $t \rightarrow 0$, we get for the derivative of the function $s \mapsto \pi(s) u(\phi)$ at $s_{0}$ along the vector $Y$

$$
Y \pi\left(s_{0}\right) u(\phi)=\pi\left(s_{0}\right) u(\tilde{Y} \phi),
$$

where the function

$$
(\tilde{Y} \phi)(x):=\lim _{t \rightarrow 0} \frac{\phi\left(y(t)^{-1} x\right)-\phi(x)}{t}
$$

belongs to $C_{0}^{\infty}(S)$. Indeed, we know from the proof of Theorem 1 in [1, Chapter 11] that $\tilde{Y} \phi \in C_{0}^{\infty}(G)$. Thus, it is sufficient to prove that the support $\operatorname{supp} \tilde{Y} \phi \subseteq \operatorname{int} S$ if $K:=\operatorname{supp} \phi \subset \operatorname{int} S$. Choose $U \subset G$ open such that $K \subset U \subset U^{-} \subset \operatorname{int} S$ ( $U^{-}$is the closure of $U$ ). Then there is the neighborhood $N$ of $e$ in $G$ with $N K \subseteq U$ and $\delta>0$ such that $y(t) \in N$ for $|t|<\delta$. Since the support of the function $x \mapsto \phi\left(y(t)^{-1} x\right)$ is $y(t) K \subseteq U$ for $|t|<\delta$, the support of the function $x \mapsto t^{-1}\left(\phi\left(y(t)^{-1} x\right)-\phi(x)\right)$ is contained in $U$, too. It follows that $\operatorname{supp} \tilde{Y} \phi \subseteq U^{-} \subset \operatorname{int} S$. Now from (2.3) we conclude that $u(\phi) \in \mathscr{V}^{\infty}$.

(ii) Suppose that $Y \in L(S)$ and $t>0$. Then the preceding arguments are true for all $s_{0} \in S$. In particular, setting in (2.2) $s_{0}=e$ and $t \rightarrow 0+$ we conclude that $u(\phi) \in$ $\mathscr{D}(A(X))$ and

$$
A(Y) u(\phi)=u(\tilde{Y} \phi)
$$


Equation (2.5) shows that $\mathscr{D}_{S}$ is $A(Y)$-invariant. The invariance of $\mathscr{D}_{S}$ with respect to $\pi(s)(s \in S)$ follows from the equality

$$
\pi(s) u(\phi)=\int_{G} \phi(x) \pi(s x) u d_{l} x=\int_{G} \phi\left(s^{-1} x\right) \pi(x) u d_{l} x .
$$

(iii) Since $e$ is an adherent point for int $S$, there is a net of compact subsets $K \subset \operatorname{int} S$, which shrinks to $e$. By an argument similar to that given in the proof of Theorem 1 in [1, Chapter 11], $\mathscr{D}_{S}$ is dense in $\mathscr{V}$.

(iv) Let $X_{1}, X_{2} \in L(S)$ and $u \in \mathscr{D}_{S}$. Then

$$
\begin{aligned}
A\left(X_{1}+X_{2}\right) u & =\left.\frac{d}{d t} \pi\left(\exp t\left(X_{1}+X_{2}\right)\right) u\right|_{t=0} \\
& =\left.\frac{d}{d t} \pi\left(\exp t X_{1} \exp t X_{2}\right)\right|_{t=0} \\
& =A\left(X_{1}\right) u+A\left(X_{2}\right) u .
\end{aligned}
$$

The proof of the equality $A\left(c X_{1}\right) u=c A\left(X_{1}\right) u(c \geq 0)$ is similar to the preceding one. Now let, by definition,

$$
\hat{A}\left(X_{1}-X_{2}\right) u:=A\left(X_{1}\right) u-A\left(X_{2}\right) u .
$$

This definition is correct, $\hat{A}$ is linear on $L(G)$ (see (1.5)) and $\hat{A} \mid L(S)=A$ on $\mathscr{D}_{S}$. The uniqueness of such an extention follows from (1.5), too.

(v) For $X \in L(S)$ and $s_{0} \in \operatorname{int} S$ the function $t \mapsto s_{0} \exp t X: \mathbb{R}^{+} \rightarrow \operatorname{int} S$ is differentiable. Therefore, for each $u \in \mathscr{V}^{\infty}$ and $t \geq 0$ the function $t \mapsto \pi\left(s_{0} \exp t X\right) u=$ $\pi\left(s_{0}\right) \pi(\exp t X) u$ is differentiable, as well. Since $\pi\left(s_{0}\right)$ is invertible, it follows that the function $t \mapsto \pi(\exp t X) u$ is differentiable at $t=0$ and so $u \in \mathscr{D}(A(X))$. The last assertion follows as in (iv).

3. Analytical vectors. The vector $u \in \mathscr{V}$ is called analytic for the representation $\pi$ if the map $s \mapsto \pi(s) u$ is analytic on $\operatorname{int} S$. Let $\mathscr{V}^{\omega}$ or $\mathscr{V}^{\omega}(\pi)$ be the space of all analytic vectors for $\pi$. It is obvious that $\mathscr{V}^{\omega} \subseteq \mathscr{V}^{\infty}$. Note that $\mathscr{V}^{\omega}$ can be trivial even for Hilbert $\mathscr{V}$ and isometric $\pi$ [3, Example 3.1.18].

Proposition 3.1. Let $S$ be a quasi-invariant generating Lie semigroup in a connected Lie group $G$ and let $\pi\left(s_{0}\right)$ be invertible for some $s_{0} \in \operatorname{int} S$. The space $\mathscr{v} \omega$ is $\pi$-invariant and $A(X)$-invariant for $X \in L(S)$.

Proof. Let $x \in S$. Since the mapping $s \mapsto s x: \operatorname{int} S \rightarrow \operatorname{int} S$ is analytic, the same is $s \mapsto \pi(s x) u=\pi(s) \pi(x) u$ for $u \in \mathscr{V}^{\omega}$. Therefore $\pi(x) u \in \mathscr{V}^{\omega}$, that is, $\mathscr{V}^{\omega}$ is $\pi(x)$ invariant.

To prove the second statement, first note that $\mathscr{V}^{\omega} \subseteq \mathscr{D}(A(X))(X \in L(S))$ by Proposition 2.3(v). Let $X \in L(S), u \in \mathcal{V}^{\omega}$, and $v:=A(X) u$. If $s \in \operatorname{int} S$, then

$$
\pi(s) v=\left.\pi(s) \frac{d}{d t} \pi(\exp t X) u\right|_{t=0}=\left.\frac{d}{d t} \pi(s \exp t X) u\right|_{t=0} .
$$

Since $s \mapsto \pi(s \exp t X) u$ is analytic on $\operatorname{int} S$, it follows that $s \mapsto \pi(s) v$ is analytic on $\operatorname{int} S$ by Vitali theorem. Indeed,

$$
\left.\frac{d}{d t} \pi(s \exp t X) u\right|_{t=0}=\lim _{h \rightarrow 0} \frac{\pi(s \exp h X) u-\pi(s) u}{h} .
$$


For sufficiently small $h$ and compact $C \subset \operatorname{int} S$ we have $(s \in C)$

$$
\left\|\frac{\pi(s \exp h X) u-\pi(s) u}{h}\right\| \leq \sup _{s \in C}\|\pi(s)\|\left\|\frac{\pi(\exp h X) u-u}{h}\right\| \leq \text { const, }
$$

because

$$
\lim _{h \rightarrow 0}\left\|\frac{\pi(\exp h X) u-u}{h}\right\|=\|A(X) u\|<\infty .
$$

Thus, the family of analytic functions $s \mapsto h^{-1}(\pi(\exp h X) u-\pi(s) u)(s \in \operatorname{int} S)$ is uniformly bounded for $s \in C$ and small $h$, and all the conditions of the Vitali theorem are satisfied.

4. The main lemma. Recall that $\hat{A}$ denotes the linear continuation from $L(S)$ to $L(G)$ of the map $A:\left.X \mapsto(d / d t) \pi(\exp t X)\right|_{t=0}$ (the right side is an operator on $\mathscr{V}^{\infty}$ whenever at least one operator $\pi(s), s \in \operatorname{int} S$, is invertible).

LEMMA 4.1. Let $S$ be a quasi-invariant generating Lie semigroup in a connected Lie group $G$ and let $\pi$ be a representation of $S$ on a Banach space $\mathscr{V}$ such that the operator $\pi\left(s_{0}\right)$ is invertible for some $s_{0} \in \operatorname{int} S$. Then $\hat{A}$ is a representation of the Lie algebra $L(G)$ by operators on $\mathscr{D}_{S}$ or $\mathscr{V}^{\omega} \omega$.

Proof. There exists a star-shaped neighborhood $B_{0} \subset L(G)$ of 0 such that $s_{0} \exp r X$ $\in \operatorname{int} S$ for $X \in B_{0}$ and $|r| \leq 2$. Fix vectors $u \in \mathscr{D}_{S}$ (or $\mathscr{q}^{\omega}$ ) and $X \in B_{0} \cap L(S)$. Applying the Taylor formula

$$
f(1)=f(0)+f^{\prime}(0)+\frac{1}{2 !} f^{\prime \prime}(0)+\frac{1}{2 !} \int_{0}^{1} f^{\prime \prime \prime}(\xi)(1-\xi)^{2} d \xi
$$

to the smooth function $f(r)=\pi\left(s_{0} \exp r X\right) u(|r| \leq 2)$, we have

$$
\begin{aligned}
\pi\left(s_{0} \exp X\right) u= & \pi\left(s_{0}\right) u+\left.\frac{d}{d r} \pi\left(s_{0} \exp r X\right) u\right|_{r=0}+\left.\frac{1}{2} \frac{d^{2}}{d r^{2}} \pi\left(s_{0} \exp r X\right) u\right|_{r=0} \\
& +\left.\frac{1}{2} \int_{0}^{1} \frac{d^{3}}{d r^{3}} \pi\left(s_{0} \exp r X\right) u\right|_{r=\xi}(1-\xi)^{2} d \xi
\end{aligned}
$$

Since for all $k \in \mathbb{N}$ and $r \geq 0$

$$
\frac{d^{k}}{d r^{k}} \pi\left(s_{0} \exp r X\right) u=\pi\left(s_{0}\right) \pi(\exp r X)(A(X))^{k} u
$$

and $\pi\left(s_{0}\right)$ is invertible, it follows from (4.2) that

$$
\pi(\exp X) u=u+A(X) u+\frac{1}{2}(A(X))^{2} u+\frac{1}{2} \int_{0}^{1} \pi(\exp \xi X)(A(X))^{3} u(1-\xi)^{2} d \xi .
$$

Now fix $X_{1}, X_{2} \in L(S)$ and choose $\delta>0$ such that $t X_{1}, t X_{2}$, and $t X_{1} * t X_{2}$ belong to $B_{0} \cap B$ for $|t|<\delta$, where $B$ is a Campbell-Hausdorff-neighborhood in $L(G)$ and (1.3) holds. Then $t X_{1} * t X_{2} \in L(S)$ for $t \in[0, \delta)$ and the Campbell-Hausdorff formula implies that

$$
t X_{1} * t X_{2}=t\left(X_{1}+X_{2}\right)+\frac{1}{2} t^{2}\left[X_{1}, X_{2}\right]+t^{3} H_{3}\left(X_{1}, X_{2}\right)+\cdots .
$$


As $\exp \left(t X_{1} * t X_{2}\right)=\exp t X_{1} \exp t X_{2}$, we have for $t \in[0, \delta), u \in \mathscr{D}_{S}$

$$
\pi\left(\exp t X_{1}\right) \pi\left(\exp t X_{2}\right) u=\pi\left(\exp \left(t X_{1} * t X_{2}\right)\right) u .
$$

If we substitute $X=t X_{1} * t X_{2}(t \in[0, \delta))$ to (4.4), then

$$
\begin{aligned}
\pi\left(\exp \left(t X_{1} * t X_{2}\right)\right) u= & u+A\left(t X_{1} * t X_{2}\right) u+\frac{1}{2}\left(A\left(t X_{1} * t X_{2}\right)\right)^{2} u \\
& +\frac{1}{2} \int_{0}^{1} \pi\left(\exp \xi\left(t X_{1} * t X_{2}\right)\right)\left(A\left(t X_{1} * t X_{2}\right)\right)^{3} u(1-\xi)^{2} d \xi .
\end{aligned}
$$

In view of (4.5) and by the continuity of the linear map $X \mapsto \hat{A}(X) u$,

$$
\begin{aligned}
A\left(t X_{1} * t X_{2}\right) u & =t\left(A\left(X_{1}\right)+A\left(X_{2}\right)\right) u+\frac{1}{2} t^{2} \hat{A}\left(\left[X_{1}, X_{2}\right]\right) u+t^{3} \hat{A}\left(H_{3}\left(X_{1}, X_{2}\right)\right) u+\cdots \\
& =\left(t\left(A\left(X_{1}\right)+A\left(X_{2}\right)\right)+\frac{1}{2} t^{2} \hat{A}\left(\left[X_{1}, X_{2}\right]\right)+t^{3} R_{1}\left(t, X_{1}, X_{2}\right)\right) u .
\end{aligned}
$$

Thus,

$$
\begin{gathered}
\left(A\left(t X_{1} * t X_{2}\right)\right)^{2} u=\left(t^{2}\left(A\left(X_{1}\right)+A\left(X_{2}\right)\right)^{2}+t^{3} R_{2}\left(t, X_{1}, X_{2}\right)\right) u, \\
\left(A\left(t X_{1} * t X_{2}\right)\right)^{3} u=t^{3} R_{3}\left(t, X_{1}, X_{2}\right) u,
\end{gathered}
$$

where the functions $t \mapsto R_{n}\left(t, X_{1}, X_{2}\right) u(n=1,2,3)$ are bounded for $t \rightarrow 0$. From (4.7) it follows, and since (4.8), (4.9), that for $t \in[0, \delta)$

$$
\begin{aligned}
\pi\left(\exp \left(t X_{1} * t X_{2}\right)\right) u= & u+t\left(A\left(X_{1}\right)+A\left(X_{2}\right)\right) u+\frac{1}{2} t^{2}\left(\hat{A}\left(\left[X_{1}, X_{2}\right]\right)+\left(A\left(X_{1}\right)+A\left(X_{2}\right)\right)^{2}\right) u \\
& +\frac{1}{2} t^{3} \int_{0}^{1} \pi\left(\exp \xi\left(t X_{1} * t X_{2}\right)\right) R_{3}\left(t, X_{1}, X_{2}\right) u(1-\xi)^{2} d \xi \\
= & u+t\left(A\left(X_{1}\right)+A\left(X_{2}\right)\right) u+\frac{1}{2} t^{2}\left(\hat{A}\left(\left[X_{1}, X_{2}\right]\right)\right. \\
& \left.+\left(A\left(X_{1}\right)+A\left(X_{2}\right)\right)^{2}\right) u+o\left(t^{2}\right),
\end{aligned}
$$

because $\left\|\pi\left(\exp \xi\left(t X_{1} * t X_{2}\right)\right)\right\|$ is bounded for $t$ sufficiently small and $\xi \in[0,1]$ by the uniform boundedness principle.

By (4.6) and $u \in \mathscr{D}_{S}$, the left-hand side in (4.10) is a smooth function of $t$. By the uniqueness of the Taylor polynomial, (4.10) implies

$$
\left.\frac{d^{2}}{d t^{2}} \pi\left(\exp \left(t X_{1} * t X_{2}\right)\right) u\right|_{t=0}=\hat{A}\left(\left[X_{1}, X_{2}\right]\right) u+\left(A\left(X_{1}\right)+A\left(X_{2}\right)\right)^{2} u \text {. }
$$

On the other hand, let

$$
F(t) u=\pi\left(\exp t X_{1}\right) \pi\left(\exp t X_{2}\right) u, \quad t \geq 0, u \in \mathscr{D}_{S} .
$$

Then, for each $\Delta t>0$

$$
\begin{aligned}
\Delta F(t) u= & \pi\left(\exp t X_{1}\right) \pi\left(\exp \Delta t X_{1}\right) \pi\left(\exp t X_{2}\right) \pi\left(\exp \Delta t X_{2}\right) u \\
& -\pi\left(\exp t X_{1}\right) \pi\left(\exp t X_{2}\right) u \\
= & \pi\left(\exp t X_{1}\right)\left(\pi\left(\exp \Delta t X_{1}\right) \pi\left(\exp \Delta t X_{2}\right) v-v\right),
\end{aligned}
$$

where $v:=\pi\left(\exp t X_{2}\right) u \in \mathscr{D}_{S}(\pi(\exp t X)$ commutes with $\pi(\exp s X)$ for all $X \in L(S)$, $t, s \geq 0$ ). 
Therefore,

$$
\begin{aligned}
\frac{d}{d t} F(t) u & =\lim _{\Delta t \rightarrow 0} \pi\left(\exp t X_{1}\right)\left(\pi\left(\exp \Delta t X_{1}\right) \frac{\pi\left(\exp \Delta t X_{2}\right) v-v}{\Delta t}+\frac{\pi\left(\exp \Delta t X_{1}\right) v-v}{\Delta t}\right) \\
& =\pi\left(\exp t X_{1}\right)\left(A\left(X_{2}\right) v+A\left(X_{1}\right) v\right) \\
& =\pi\left(\exp t X_{1}\right) A\left(X_{1}\right) \pi\left(\exp t X_{2}\right) u+\pi\left(\exp t X_{1}\right) A\left(X_{2}\right) \pi\left(\exp t X_{2}\right) u
\end{aligned}
$$

Since $\pi\left(\exp t X_{i}\right)$ commutes with $A\left(X_{i}\right), i=1,2$,

$$
\frac{d}{d t} F(t) u=A\left(X_{1}\right) F(t) u+F(t) A\left(X_{2}\right) u .
$$

Because $A\left(X_{1}\right)$ is close, it follows that

$$
\begin{aligned}
\left.\frac{d^{2}}{d t^{2}} F(t) u\right|_{t=0} & =A\left(X_{1}\right)\left(A\left(X_{1}\right) u+A\left(X_{2}\right) u\right)+A\left(X_{1}\right) A\left(X_{2}\right) u+A\left(X_{2}\right) A\left(X_{2}\right) u \\
& =\left(A\left(X_{1}\right)\right)^{2} u+2 A\left(X_{1}\right) A\left(X_{2}\right) u+\left(A\left(X_{2}\right)\right)^{2} u .
\end{aligned}
$$

Now differentiating (4.6) twice at $t=0$ we obtain for $X_{1}, X_{2} \in L(S)$ by virtue of (4.11)

$$
\left(A\left(X_{1}\right)\right)^{2} u+2 A\left(X_{1}\right) A\left(X_{2}\right) u+\left(A\left(X_{2}\right)\right)^{2} u=\hat{A}\left(\left[X_{1}, X_{2}\right]\right) u+\left(A\left(X_{1}\right)+A\left(X_{2}\right)\right)^{2} u,
$$

or

$$
\hat{A}\left(\left[X_{1}, X_{2}\right]\right) u=\left[\hat{A}\left(X_{1}\right), \hat{A}\left(X_{2}\right)\right] u .
$$

Since both sides of the last equality are bilinear, it is valid for all $X_{1}, X_{2} \in L(G)$ by (1.5). This completes the proof.

EXAMPLE 4.2. Let $S$ be as in Lemma 4.1 and $\mathscr{F}$ a finite-dimensional subspace of $L^{p}(S)(1 \leq p<\infty)$ which is invariant under left translations $\lambda(s) f(x)=f(s x)(s, x \in$ $S)$. Then $\lambda$ is a left regular representation of $S$ on $\mathscr{F}$. Since $t \mapsto \lambda(t \exp X)(t \geq 0, X \in$ $L(S))$ is a one-parameter $C_{0}$-semigroup on $\mathscr{F}$, the operator $\lambda(\exp X)$ is invertible. Thus $\lambda(s)$ is invertible for all $s \in\langle\exp L(S)\rangle \supseteq \operatorname{int} S$ and $d \lambda$ extends to a representation $\hat{A}$ of $L(G)$ on $\mathscr{F}$ by Lemma 4.1. Then the local representation $T$ of $G$ on $\mathscr{F}$ with $d T=\hat{A}$ extends $\lambda$. The representation $T$ is global for simply connected $G$.

5. Extension of unitary representations. Our first statement deals with local extensions.

THEOREM 5.1. Let $\pi$ be a unitary representation on a Hilbert space $\mathcal{H}$ of a quasiinvariant generating Lie semigroup $S$ in a connected Lie group $G$ and let one of the following conditions holds:

(1) $\mathscr{H}^{\mathrm{\omega}}$ is dense in $\mathscr{H}$;

(2) for some linear independent $X_{1}, \ldots, X_{d} \in L(S)(d=\operatorname{dim} L(G))$ the Nelson operator

$$
\Delta=\sum_{j=1}^{d}\left(A\left(X_{j}\right)\right)^{2}
$$

is essentially selfadjoint on $\mathscr{D}_{S}$. 
Then $\pi$ extends to a local unitary representation of $G$ on H. Moreover, if $S$ algebraically generates $G$, for some e-neighborhood $U \subset G$ the extension of $\pi$ to a local representation of $U$ is unique.

PROof. (1) First, prove that every vector $u \in \mathscr{H}^{\omega}$ is analytic for the operator $A(X)$ if $X \in L(S)$ satisfies $\exp X \in \operatorname{int} S$. Indeed, $\mathscr{H}^{\omega} \subseteq \mathscr{D}(A(X))$ and since the function $t \mapsto$ $\exp t X: \mathbb{R} \rightarrow G$ is analytic, the function $t \mapsto \pi(\exp t X) u$ has the same property in the same $\epsilon$-neighborhood of the point $t=1$. Therefore, $u$ is an analytic vector of the one-parameter unitary group $T_{1}(t)$ which agrees with $\pi(\exp t X)$ for $t \geq 0$ and has the generator $A(X)$ (in the sense of semigroup theory). Standard Cauchy estimates for the $\operatorname{disc}\{|t-1|<\epsilon\}$ give

$$
\left\|(A(X))^{n} u\right\|=\left\|T_{1}(1)(A(X))^{n} u\right\|=\left\|\left.\frac{d^{n}}{d t^{n}} T_{1}(t) u\right|_{t=1}\right\| \leq \frac{n ! M}{\epsilon^{n}}
$$

for some $M>0$, and so $u$ is an analytic vector for $A(X)$.

Being the generator of $T_{1}(t)$, operator $A(X)$ is antiselfadjoint and so, it is antisymmetric on $\mathscr{H}^{\omega}(X \in L(S))$. We claim that $\exp X_{j} \in \operatorname{int} S$ for some linear independent $X_{j} \in L(S), j=1, \ldots, d$. Since $L(S)$ is generating in $L(G)$, zero is in the closure of int $L(S)$ (with respect to $L(G)$ ). Let $B_{1}$ be a neighborhood of zero in $L(G)$ such that $\left.\exp \right|_{B_{1}}$ is a homomorphism on its image. Then $\exp \left(B_{1} \cap \operatorname{int} L(S)\right)$ is a nonvoid open subset of $S$, and we may choose linear independent $X_{j} \in B_{1} \cap \operatorname{int} L(S), j=1, \ldots, d$.

Now if (1) holds, then for the representation $\hat{A}$ of the Lie algebra $L(G)$ by operators on $\mathscr{H}^{\omega}$ (see Lemma 4.1) all the conditions of the $F S^{3}$-criterion (cf. [1, Chapter 11, Section 6, Theorem 5]), except the simply connectedness of $G$, are satisfied. But the proof of the $F S^{3}$-criterion in [1] shows that for connected $G$ we get a unitary representation $T$ of a local group $W$, where $W$ is a neighborhood of unit in $G$, and a $T$-invariant dense linear subspace $\mathscr{H}_{\infty} \subseteq \mathscr{H}$ such that for all $X \in L(G)$ we have $\mathscr{H}^{\omega} \subseteq \mathscr{H}_{\infty} \subseteq \mathscr{D}(T(X))$ and $d T(X)\left|\mathscr{H}_{\infty}=\overline{\hat{A}(X)}\right| \mathscr{H}_{\infty}$ (the bar denotes the closure of an operator; see Lemmas 3,4 , and formula (11) therein). Since the one-parameter $C_{0}$-semigroup $T_{1}(t)=\pi(\exp t X), X \in L(S)$, leaves $\mathscr{H}^{\omega} \omega$ invariant, $\mathscr{H}^{\omega}$ is an essential domain for $A(X)$ by [3, Corollary 3.1.7]. Hence, $\overline{\hat{A}(X)}=\overline{A(X) \mid \mathscr{H}^{\omega}}=A(X)$ for $X \in L(S)$, and the definition of $\mathscr{H}_{\infty}$ in [1, Chapter 11, Section 6, Lemma 3] entails that $\mathscr{H}_{\infty} \subseteq \mathscr{D}(A(X))$. The first assertion follows now from the next proposition.

Proposition 5.2. Let $\pi$ be a representation on a Banach space $\mathcal{V}$ of a quasiinvariant generating Lie semigroup $S$ in a connected Lie group $G$.

(i) Let $T$ be a local representation of $G$ on $\mathscr{V}$ and let $\mathscr{V}_{0} \subseteq \mathscr{V}$ be a $T$-invariant dense linear subspace such that $\mathscr{V}_{1} \subseteq \mathscr{V}_{0} \subseteq \mathscr{D}(T(X)) \cap \mathscr{D}(A(X))$ for a some $\pi$-invariant dense linear subspace $\mathscr{V}_{1} \subseteq \mathscr{V}$ and $d T(X)\left|\mathscr{V}_{0}=A(X)\right| \mathscr{V}_{0}$ for all $X \in L(S)$. Then $T \mid U \cap S=$ $\pi \mid U \cap S$ for some e-neighborhood $U \subseteq G$, and $\pi(s)$ is invertible for all $s \in S$.

(ii) If $S$ algebraically generates $G$, then for some e-neighborhood $U \subseteq G$ the extension of $\pi$ to a local representation of $U$ (if such exists) is unique.

Proof. (i) Let $T$ be a representation of a local group $W$, where $W$ is a neighborhood of $e$ in $G$. Fix $X \in L(S)$ and pick $\delta>0$ such that $\exp t X \in W$ for $t \in \mathbb{R}$ with $|t|<\delta$. The 
map $t \mapsto T(\exp t X)$ is a representation of the additive local group $(-\delta, \delta)$ and for $\delta$ sufficiently small it extends to the one-parameter group $T_{X}$ on $\mathscr{V}$ with the generator $d T(X)$ (cf. [2, Chapter 3, Section 6, Lemma 1]), and $\mathscr{V}_{0}$ is $T_{X}$-invariant. Consider also the $C_{0}$-semigroup $T_{1}(t)=\pi(\exp t X), t \in \mathbb{R}^{+}$, with generator $A(X)$. Note that generators of $T_{X}$ and $T_{1}$ coincide on $\mathscr{V}_{0}$ and that $\mathscr{V}_{0}$ and $\mathscr{V}_{1}$ are essential domains for $d T(X)$ and $A(X)$, respectively, by [3, Corollary 3.1.7]. Since $\mathscr{V}_{1} \subseteq \mathscr{V}_{0} \subseteq \mathscr{D}(A(X)), \mathscr{V}_{0}$ is an essential domain for $A(X)$, too. Therefore, $d T(X)=A(X)$ and hence $T_{X}(t)=T_{1}(t)$ for all $t \in \mathbb{R}^{+}$. In particular, $T(\exp t X)=\pi(\exp t X)$ for $0 \leq t<\delta$. Let $B_{0}$ be a star-shaped neighborhood of zero in $L(G)$ such that $\exp B_{0} \subset W$. Then the preceding equality implies that $T(\exp X)=\pi(\exp X)$ for $X \in B_{0} \cap L(S)$. But $\exp \left(B_{0} \cap L(S)\right) \supset U \cap S$ for some $e$-neighborhood $U \subset W$, because $L(S)$ is a semialgebra [15, Theorem III.9], and hence $T|U \cap S=\pi| U \cap S$.

The equality $T_{X}(t)=T_{1}(t), t \geq 0$, shows also that $\pi(\exp X)$ is invertible for $X \in$ $L(S)$. It follows that $\pi\left(s_{0}\right)$ is invertible for $s_{0} \in\langle\exp L(S)\rangle \supseteq \operatorname{int} S$, and so is $\pi(s)=$ $\pi\left(s s_{0}\right) \pi\left(s_{0}\right)^{-1}$ for all $s \in S\left(s_{0} \in \operatorname{int} S\right)$.

(ii) As before, for an arbitrary small $e$-neighborhood $U$ in $G$ we have $U \cap S=\exp (B \cap$ $L(S)$ ) ( $B \subset L(G)$ is a neighborhood of zero) by [15, Theorem III.9]. Then the set $U \cap$ $\sigma_{X}$ algebraically generates the full one-parameter semigroup $\sigma_{X}$ corresponding to $X \in L(S)$. Therefore, the set $U \cap S$ algebraically generates the semigroup $\langle\exp L(S)\rangle \supseteq$ int $S$. Being an ideal in $S$, int $S$ algebraically generates $G$, too. Thus $U \cap S$ algebraically generates $G$ and the local representation $T$ is completely determined by its restriction $T \mid U \cap S$. This completes the proof of Proposition 5.2.

(2) Let condition (2) of Theorem 5.1 be satisfied. As it was mentioned above, $A(X)$ is an antiselfadjoint operator and $\mathscr{D}_{S}$ is an essential domain for $A(X), X \in L(S)$, by [3, Corollary 3.1.7]. Therefore, operator $i A(X) \mid \mathscr{D}_{S}$ has a selfadjoint closure. Thus for the representation $\hat{A}$ of the Lie algebra $L(G)$ by operators on $\mathscr{D}_{S}$ all the conditions of the Nelson criterion (cf. [1, Chapter 11, Section 5, Theorem 2]), excepting the simply connectedness of $G$, are satisfied. The proof of this criterion (see especially Lemma 1 therein) shows that for connected $G$ we get a local unitary representation $T$ of some neighborhood $N$ of unit in $G$ such that for $X \in L(G)$ with $\exp X \in N$ we have $T(\exp X)=e^{\hat{A}(X)}:=T_{X}(1)$, where $T_{X}$ is a one-parameter unitary group on $\mathscr{H}$ with the generator $\overline{\hat{A}(X)}$ (in the sense of semigroup theory). Choose, as in the proof of Proposition 5.2, a neighborhood of zero $B_{0} \subset L(G)$ such that $\exp B_{0} \subset N$ and $\exp \left(B_{0} \cap\right.$ $L(S)$ ) contains $U \cap S$ for some $e$-neighborhood $U \subset G$. Since $\bar{A}(X)=A(X)$ for $X \in L(S)$, $T_{X}(t)=T_{1}(t):=\pi(\exp t X)$ for $X \in L(S), t \geq 0$. Thus for $X \in B_{0} \cap L(S)$ we have $T(\exp X)=\pi(\exp X)$ and $T|U \cap S=\pi| U \cap S$. In other words, $T$ is an extension of $\pi$ to $U$. The uniqueness of such an extention follows from Proposition 5.2 immediately.

We call a Lie group $G$ exponential if $\exp L(G)=G$.

THEOREM 5.3. Let $G$ be a connected exponential and solvable Lie group, $S$ a quasiinvariant generating Lie subsemigroup of $G$. A unitary representation $\pi$ of $S$ on a Hilbert space Hextends to the whole $G$ if and only if condition (2) of Theorem 5.1 and the following condition (3) hold: 
(3) if $X_{1,2}, Y_{1,2} \in L(S)$ satisfy $\exp \left(X_{1}-X_{2}\right)=\exp \left(Y_{1}-Y_{2}\right)$, then

$$
\lim _{n \rightarrow \infty}\left(\pi\left(\exp \frac{1}{n} X_{1}\right)\left(\pi\left(\exp \frac{1}{n} X_{2}\right)\right)^{-1}\right)^{n}=\lim _{n \rightarrow \infty}\left(\pi\left(\exp \frac{1}{n} Y_{1}\right)\left(\pi\left(\exp \frac{1}{n} Y_{2}\right)\right)^{-1}\right)^{n}
$$

(the strong operator convergence). Moreover, such an extension is unique.

Proof. The existence of a basis $X_{1}, \ldots, X_{d} \in L(S)$ has been proved above (Theorem 5.1 ), and the necessity of (2) follows from the Nelson-Stinespring theorem (cf. [1, Chapter 11, Section 2, Theorem 2]).

Next we have

$$
\exp \left(X_{1}-X_{2}\right)=\lim _{n \rightarrow \infty}\left(\exp \frac{1}{n} X_{1}\left(\exp \frac{1}{n} X_{2}\right)^{-1}\right)^{n},
$$

and the same formula for $\exp \left(Y_{1}-Y_{2}\right)$. The necessity of (3) follows.

Now let (2) and (3) hold. By Theorem 5.1, there is a local unitary representation $T$ which extends $\pi$ to some $e$-neighborhood $N \subseteq G$. For $X \in L(G)$ denote by $T_{X}$ the unitary one-parameter group in $\mathscr{H}$ such that $T_{X}(t)=T(\exp t X)$ for $t \in \mathbb{R}$ with $\exp t X \in N$. The proof of Theorem 5.1 yields that the antiselfadjoint operator $\bar{A}(X)$ is the generator of $T_{X}$. We extend $T$ to the full $G$ setting $\hat{\pi}(\exp X):=T_{X}(1)$. To prove the correctness of this definition one should show that $\exp X=\exp Y$ implies $T_{X}(1)=T_{Y}(1)$ for $X, Y \in L(G)$. Let $X=X_{1}-X_{2}$ where $X_{1}, X_{2} \in L(S)$. We claim that $\overline{A\left(X_{1}\right)-A\left(X_{2}\right)}=\overline{\hat{A}(X)}$ and that the Trotter multiplicative formula can be applied to the unitary group $T_{X}$ (cf. [17, Theorem VIII.31]). Indeed, by definition $\hat{A}(X)=\left(A\left(X_{1}\right)-A\left(X_{2}\right)\right) \mid \mathscr{D}_{S}$. Since the operator $A\left(X_{1}\right)-A\left(X_{2}\right) \supseteq \hat{A}(X)$ is antisymmetric, it has an antisymmetric closure $\overline{A\left(X_{1}\right)-A\left(X_{2}\right)} \supseteq \overline{\hat{A}(X)}$, and the hypermaximality property of a selfadjoint operator implies $\overline{A\left(X_{1}\right)-A\left(X_{2}\right)}=\overline{\hat{A}(X)}$, an antiselfadjoint operator. Now by the Trotter formula mentioned above

$$
\begin{aligned}
T_{X}(1) & =e^{\overline{A\left(X_{1}\right)-A\left(X_{2}\right)}}=\lim _{n \rightarrow \infty}\left(e^{(1 / n) A\left(X_{1}\right)} e^{-(1 / n) A\left(X_{2}\right)}\right)^{n} \\
& =\lim _{n \rightarrow \infty}\left(\pi\left(\exp \frac{1}{n} X_{1}\right)\left(\pi\left(\exp \frac{1}{n} X_{2}\right)\right)^{-1}\right)^{n},
\end{aligned}
$$

so the mapping $\hat{\pi}$ is well defined by virtue of (3).

Let $G_{1}$ be the simply connected Lie group with $L\left(G_{1}\right)=L(G)$. Then there exist $e$ neighborhoods $U \subseteq N$ and $U_{1} \subseteq G_{1}$ and a local Lie group morphism $\varphi: U \rightarrow U_{1}$ such that $\varphi$ and its reciprocal $\varphi^{-1}$ both are analytic. Write $\pi_{1}(x):=T\left(\varphi^{-1}(x)\right), x \in U_{1}$, and extend $\pi_{1}$ to a (unique) unitary representation $\pi_{1}$ of $G_{1}$ on $\mathscr{H}$. Then for each $X \in L(G)$ and sufficiently large $n \in \mathbb{N}$ we have

$$
\begin{aligned}
\hat{\pi}(\exp X) & =T_{X}(1)=\left(T_{X}\left(\frac{1}{n}\right)\right)^{n}=\left(T\left(\exp \frac{1}{n} X\right)\right)^{n} \\
& =\left(\pi_{1}\left(\varphi\left(\exp \frac{1}{n} X\right)\right)\right)^{n}=\pi_{1}\left(\left(\varphi\left(\exp \frac{1}{n} X\right)\right)^{n}\right)
\end{aligned}
$$

Hence, the mapping $f(X):=\hat{\pi}(\exp X) v$ is analytic on $L(G)$ for every $v$ in $\mathscr{H}^{\omega}\left(\pi_{1}\right)$, which is a $\pi_{1}$-invariant dense linear subspace of $\mathscr{H}$, and thus the map $(X, Y) \mapsto$ 
$\hat{\pi}(\exp X) \hat{\pi}(\exp Y) v$ is separately analytic on $L(G) \times L(G)$ (formula (5.6) shows that ${ }^{\omega} \omega\left(\pi_{1}\right)$ is $\hat{\pi}(\exp X)$-invariant).

Since $G$ is exponential and solvable, for each $x_{0} \in G$ there is an $X_{0} \in L(G)$ such that $x_{0}=\exp X_{0}$ and exp is regular at $X_{0}$ [18, Theorem IV.2.44] (see also [4]). So the mapping $\exp ^{-1}=\log$ is analytic on the neighborhood $U:=\exp B$ of $x_{0}$ for some neighborhood $B$ of $X_{0}$ in $L(G)$. Therefore the function $x \mapsto \hat{\pi}(x) v=f(\log x)(x \in U)$ is analytic for $v \in$ $\mathscr{H}^{\omega}\left(\pi_{1}\right)$ too, and hence the map $(X, Y) \mapsto \hat{\pi}(\exp X \exp Y) v$ is analytic on $L(G) \times L(G)$.

Because $T$ is a local representation, the equality

$$
\hat{\pi}(\exp X \exp Y) v=\hat{\pi}(\exp X) \hat{\pi}(\exp Y) v
$$

holds for all $X, Y \in L(G)$ with sufficiently small norms, and $v \in \mathscr{H}^{\omega}\left(\pi_{1}\right)$. The separate analyticity of both sides of (5.7) implies that this formula is valid for all $X, Y \in L(G)$, $v \in \mathscr{H}^{\omega}\left(\pi_{1}\right)$. By continuity, (5.7) holds for all $v \in \mathscr{H}$ so $\hat{\pi}$ is a unitary representation of $G$ (the continuity of $\hat{\pi}$ on $G$ is a consequence of its continuity on $N$ ). The application of Corollary 2.2 shows that $\hat{\pi}$ is an extension of $\pi$ to $G$.

Finally, let $\pi^{\prime}$ be a unitary extension of $\pi$ to $G$. Note that $\mathscr{H}^{\omega}\left(\pi^{\prime}\right) \subseteq \mathcal{H}^{\omega}(\pi)$. Since for all $X_{1}, X_{2} \in L(S), u \in \mathscr{H}^{\omega}\left(\pi^{\prime}\right)$,

$$
d \pi^{\prime}\left(X_{1}-X_{2}\right) u=d \pi^{\prime}\left(X_{1}\right) u-d \pi^{\prime}\left(X_{2}\right) u=d \pi\left(X_{1}\right) u-d \pi\left(X_{2}\right) u
$$

and (1.5) hold, $\pi^{\prime}$ is completely determined by $\pi$, which completes the proof.

6. The case of Banach representations. We begin with a very simple case when $G$ is the left quotient group for $S$ [10, Chapter 1, Section 1.10], that is, $S \subset G$ and $G=S^{-1} S$.

Proposition 6.1. Let $G$ be a topological group and a group of left quotients for $S$, int $S \neq \varnothing$. The representation $\pi$ of $S$ on a Banach space $\mathscr{V}$ is extendable to a representation of $G$ on $\mathscr{V}$ if and only if all operators $\pi(s), s \in S$, are invertible. Moreover, such an extension is unique.

PROoF. To prove the sufficiency, for every $x \in G, x=a^{-1} b(a, b \in S)$, set $\hat{\pi}(x):=$ $\pi(a)^{-1} \pi(b)$. Let, in addition, $x=c^{-1} d(c, d \in S)$. Then $a c^{-1}=p^{-1} q$ for some $p, q \in$ $S$. Since $p a=q c, \pi(p) \pi(a)=\pi(q) \pi(c)$. On the other hand, $a^{-1} b=c^{-1} d$ implies $p b=q d$. Therefore, $\pi(p) \pi(b)=\pi(q) \pi(d)$ or

$$
\pi(p) \pi(a) \hat{\pi}\left(a^{-1} b\right)=\pi(q) \pi(c) \hat{\pi}\left(c^{-1} d\right) .
$$

Thus, we proved that $\hat{\pi}\left(a^{-1} b\right)=\hat{\pi}\left(c^{-1} d\right)$ and the definition of $\hat{\pi}$ is consistent.

Now let $x=a^{-1} b$ and $y=c^{-1} d(a, b, c, d \in S)$ are arbitrary elements of $G$, and let $b c^{-1}=r^{-1} s$, where $r, s \in S$. Then $x y=(r a)^{-1} s d$ and we have

$$
\hat{\pi}(x y)=(\pi(r a))^{-1} \pi(s d)=\pi(a)^{-1} \pi(r)^{-1} \pi(s) \pi(d) .
$$

Since $r b=s c, \pi(r)^{-1} \pi(s)=\pi(b) \pi(c)^{-1}$. Therefore

$$
\hat{\pi}(x y)=\pi(a)^{-1} \pi(b) \pi(c)^{-1} \pi(d)=\hat{\pi}(x) \hat{\pi}(y) .
$$

Finally, $\hat{\pi}$ is continuous on $G$, because it is continuous on int $S$. The uniqueness of $\hat{\pi}$ is obvious. 
COROLlARY 6.2. Let $S$ be a generating Lie subsemigroup in a connected Lie group $G$ and let one of the following conditions be satisfied:

(1) $G$ is nilpotent.

(2) $L(G)$ is a compact Lie algebra.

(3) $S$ is invariant in $G$.

Then every representation of $S$ by invertible operators on a Banach space $\mathcal{V}$ extends uniquely to the representation of $G$ on $\mathscr{V}$.

Indeed, by [7, Theorem 3.46], $G$ is a left quotient group for $S$.

The general case is more complicated. The following theorem describes the tangent map of a Banach representation of $S$ which extends to a local representation of $G$. We assume below that $\mathscr{D}(\hat{A}(X))=\mathscr{D}_{S}$.

THEOREM 6.3. Let $S$ be a quasi-invariant generating Lie subsemigroup in a connected Lie group $G$. The representation $\pi$ of $S$ on a Banach space $\mathscr{V}$ is extendable to a local representation of $G$ on $\mathscr{V}$ which leaves $\mathscr{D}_{S}$ invariant if and only if the following conditions hold:

(a) the operators $\hat{A}(X)$ are closable for all $X \in L(G)$;

(b) there exist constants $C$ and $\epsilon>0$ such that for all $X \in L(G)$ with the norms $|X|<1$ and $|\operatorname{Re} \lambda|>\epsilon$ the resolvents $R(\lambda, \hat{A}(X))$ are defined and

$$
\left\|R^{n}(\lambda, \hat{A}(X))\right\| \leq \frac{C}{(|\operatorname{Re} \lambda|-\epsilon)^{n}}, \quad n \in \mathbb{N} ;
$$

(c) $\mathscr{D}_{S}$ is invariant with respect to $R(\lambda, \hat{A}(X)), X \in L(G)$.

In this case, all operators $\pi(s), s \in S$, are invertible. Moreover, if $S$ algebraically generates $G$, for some e-neighborhood $U$ in $G$ the extension of $\pi$ to a local representation of $U$ is unique.

Proof. Let $T$ be a representation of a local Lie group $U \subset G$ ( $U$ is a neighborhood of unit) which extends $\pi$ and leaves $\mathscr{D}_{S}$ invariant. The fact that $T|U \cap S=\pi| U \cap S$ entails that $d T(X) u=\hat{A}(X) u(=A(X) u)$ for all $X \in L(S), u \in \mathscr{D}_{S}$. In view of formula (1.5), the last equality is valid for all $X \in L(G)$ by linearity, and conditions (a), (b), (c) follow from the Krein-Shihvatov theorem [11] in the Kirillov formulation [9, Section 10.5, Theorem 2], for $\mathscr{V}^{0}=\mathscr{D}_{S}$.

Now let $\pi$ satisfies (a), (b), and (c). We claim that $\pi(s)$ is invertible for each $s \in S$. Indeed, since $\mathscr{D}_{S}$ is an essential domain for $A(X), X \in L(S)$, condition (b) implies that $A(X)$ is a generator of a $C_{0}$-group by Gelfand theorem [5]. Thus operators $\pi(\exp t X)$, $t \geq 0$, are invertible and the invertibility of $\pi(s)$ follows as in Proposition 5.2. According to Lemma 4.1, $\hat{A}$ is a representation of $L(G)$ by operators on $\mathscr{D}_{S}$. Again, by the Krein-Shihvatov theorem in the Kirillov formulation for $\mathscr{V}^{0}=\mathscr{D}_{S}, T^{0}=\hat{A}$, there exists a representation $T$ of some local Lie group $W$ with $L(W)=L(G)$ such that $d T(X)$ | $\mathscr{D}_{S}=\hat{A}(X), X \in L(G)$, and $\mathscr{D}_{S}$ is $T$-invariant. After shrinking $W$ we may assume that $W$ is an $e$-neighborhood in $G$ and then apply Proposition 5.2 with $\mathscr{V}_{0}=\mathscr{V}_{1}=\mathscr{D}_{S}$.

COROLLARY 6.4. Let $S$ be a quasi-invariant generating Lie semigroup in a simply connected Lie group $G$. The representation $\pi$ of $S$ on a Banach space $\mathcal{V}$ extends to a representation of the full $G$ on $\mathscr{V}$ which leaves $D_{S}$ invariant if and only if conditions (a), (b), and (c) of Theorem 6.3 hold. Moreover, such an extension is unique. 
Proof. To demonstrate the sufficiency, note that for simply connected $G$ the local representation $T$ constructed in Theorem 6.3 extends uniquely to the representation $\hat{\pi}$ of the whole $G$. Since every symmetric $e$-neighborhood generates $G$ as a semigroup, the extension $\hat{\pi}$ leaves $\mathscr{D}_{S}$ invariant, as well. Finally, $\hat{\pi} \mid S=\pi$ by Lemma 2.1.

The uniqueness of the extension can be proved as in Theorem 5.3. This completes the proof.

REMARK 6.5. The results in Sections 4,5 , and 6 are true with any $A(X)$ - and $\pi$ invariant dense linear subspace $\mathscr{D} \subseteq \mathscr{V}^{\infty} \cap \mathscr{D}(A(X)), X \in L(S)$, in place of $\mathscr{D}_{S}$.

\section{REFERENCES}

[1] A. O. Barut and R. Raczka, Theory of Group Representations and Applications, PWNPolish Scientific Publishers, Warsaw, 1977.

[2] N. Bourbaki, Groupes et Algèbras de Lie, Masson, Paris, 1990 (French).

[3] O. Bratteli and D. W. Robinson, Operator Algebras and Quantum Statistical Mechanics. Vol. 1, Springer-Verlag, New York, 1979.

[4] D. Z. Dokovic and K. H. Hofmann, The exponential map in real Lie algebras, J. Lie Theory 7 (1997), 177-199.

[5] I. Gelfand, On one-parametrical groups of operators in a normed space, CR (Doklady) Acad. Sci. URSS (NS) 25 (1939), 713-718.

[6] J. Hilgert, K. H. Hofmann, and J. D. Lawson, Lie Groups, Convex Cones, and Semigroups, Oxford Mathematical Monographs, The Clarendon Press Oxford University Press, New York, 1989, Oxford Science Publications.

[7] J. Hilgert and K. H. Neeb, Lie Semigroups and Their Applications, Lecture Notes in Math., vol. 1552, Springer-Verlag, Berlin, 1993.

[8] K. H. Hofmann and J. D. Lawson, Foundations of Lie semigroups, Recent Developments in the Algebraic, Analytical, and Topological Theory of Semigroups (Oberwolfach, 1981), Lecture Notes in Math., vol. 998, Springer, Berlin, 1983, pp. 128-201.

[9] A. A. Kirillov, Elements of Representation Theory, Springer-Verlag, Berlin, 1976.

[10] A. Klifford and G. Preston, Algebraicheskaya Teoriya Polugrupp. Tom 1 [The Algebraic Theory of Semigroups. Vol. 1], Izdat. "Mir", Moscow, 1972 (Russian), translated from the English by V. A. Baranovskii and V. G. Zitomirskii. Edited by L. N. Sevrin.

[11] S. G. Krein and A. M. Shihvatov, Linear differential equations on a Lie group, Funkcional. Anal. i Priložen. 4 (1970), no. 1, 52-61.

[12] A. R. Mirotin, On the infinite-dimensional representations of Lie semigroups, preprint 89, Skoryna Gomel State University, 1999.

[13] _ Positive semicharacters of Lie semigroups, Positivity 3 (1999), no. 1, 23-31.

[14] _ On the extension of finite-dimensional representations of Lie semigroups, Vestsī Nats. Akad. Navuk Belarusī Ser. Fìz.-Mat. Navuk (2001), no. 1, 18-21, 140.

[15] K. H. Neeb, On the foundations of Lie semigroups, J. Reine Angew. Math. 431 (1992), 165-189.

[16] _ Invariant subsemigroups of Lie groups, Mem. Amer. Math. Soc. 104 (1993), no. 499, viii+193.

[17] M. Reed and B. Simon, Methods of Modern Mathematical Physics. IV. Analysis of Operators, Academic Press, New York, 1978.

[18] M. Wustner, Contributions to the Structure Theory of Solvable Lie Groups, Dissertation (German). Technische Hochschule Darmstadt, Germany, 1995.

AdOLF R. Mirotin: DePARTMENT OF MATHEMATICS, GOMEL STATE UNIVERSITY, SOVIETSKAYA, 104, 246699 GOMEL, BELARUS

E-mail address: ami rotin@gsu. unibe1 . by 


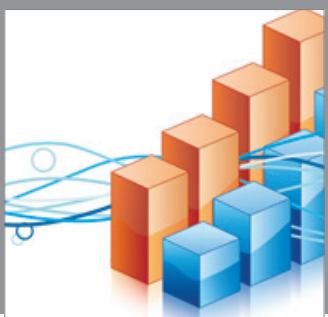

Advances in

Operations Research

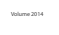

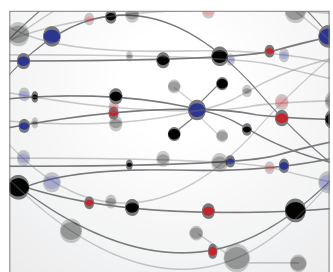

\section{The Scientific} World Journal
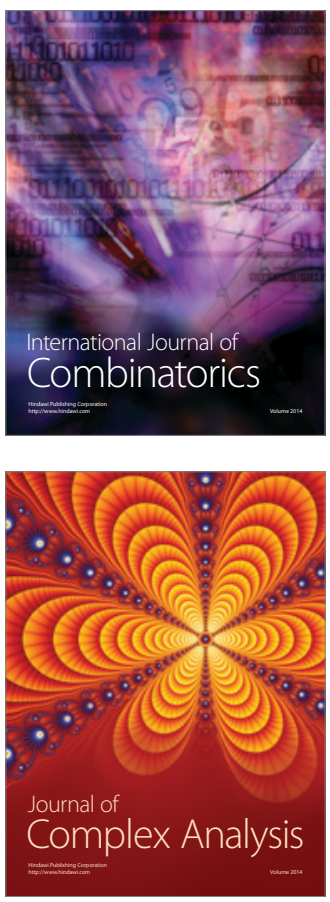

International Journal of

Mathematics and

Mathematical

Sciences
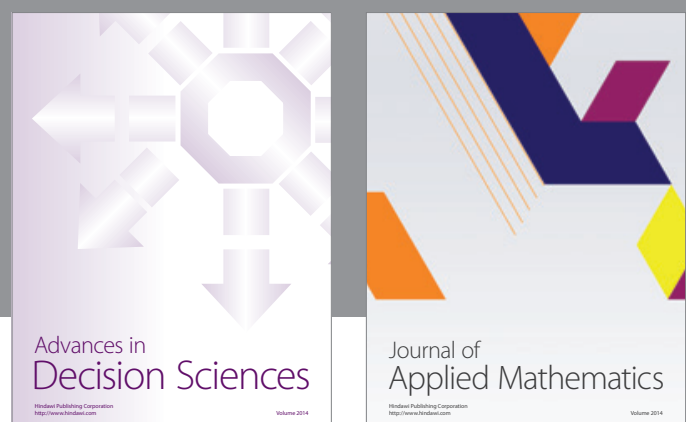

Journal of

Applied Mathematics
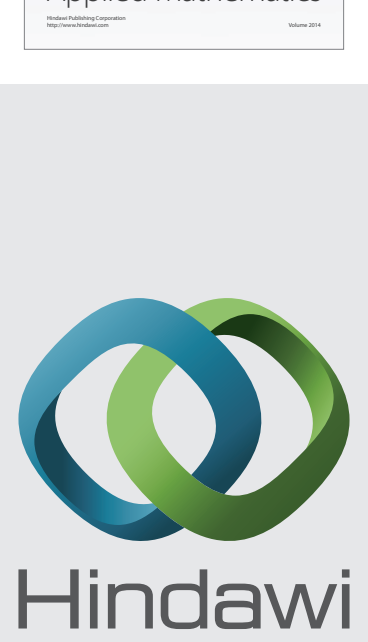

Submit your manuscripts at http://www.hindawi.com
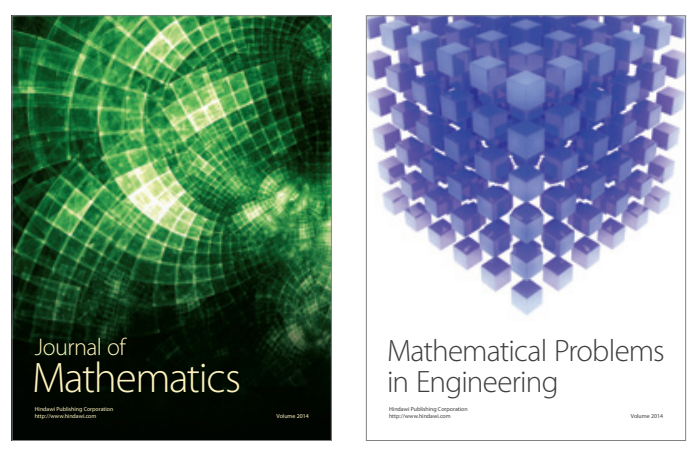

Mathematical Problems in Engineering
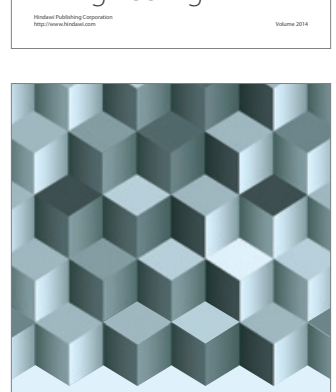

Journal of

Function Spaces
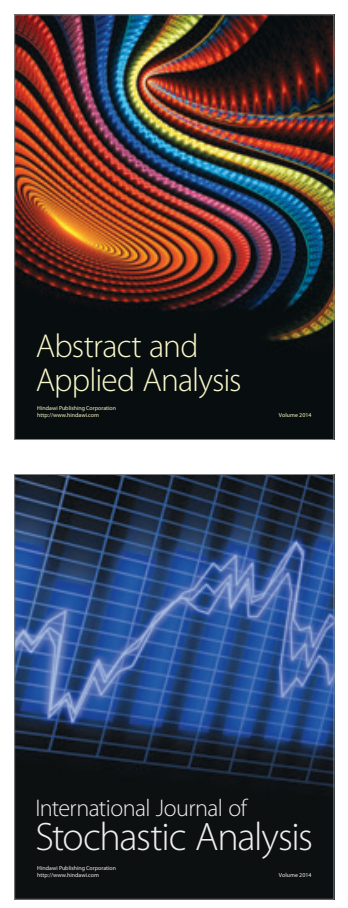

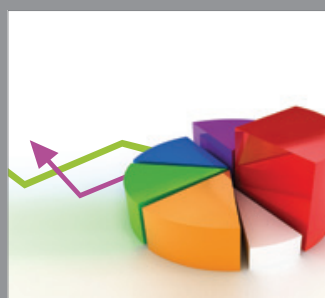

ournal of

Probability and Statistics

Promensencen
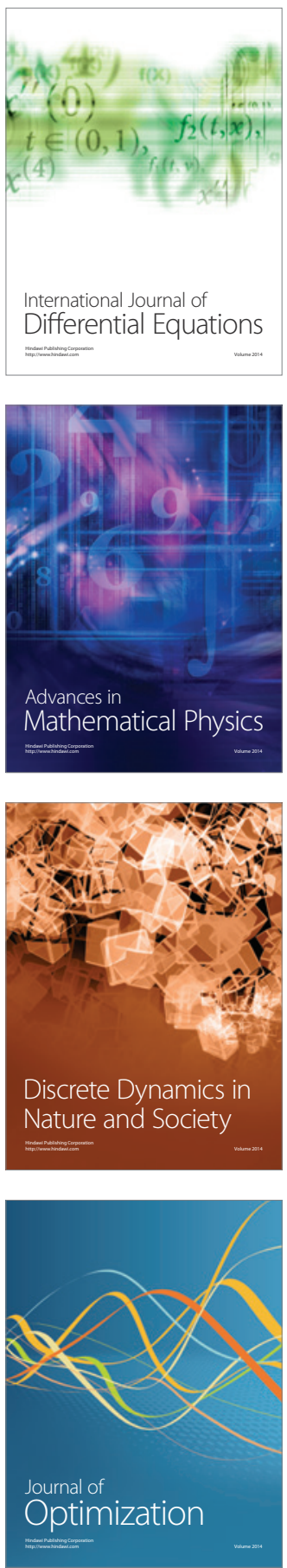